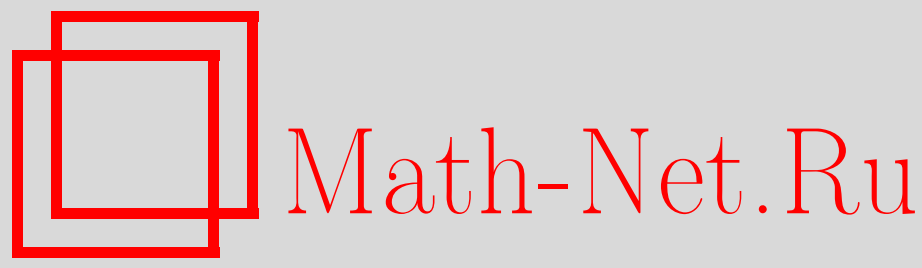

М. А. Соловьев, Некоммутативные деформации квантовых теорий поля, локальность и причинность, ТMФ, 2010, том 163, номер 3, 413-429

DOI: https://doi.org/10.4213/tmf6511

Использование Общероссийского математического портала Math-Net.Ru подразумевает, что вы прочитали и согласны с пользовательским соглашением http://www.mathnet.ru/rus/agreement

Параметры загрузки:

IP: 54.198 .55 .26

26 апреля 2023 г., 15:05:38

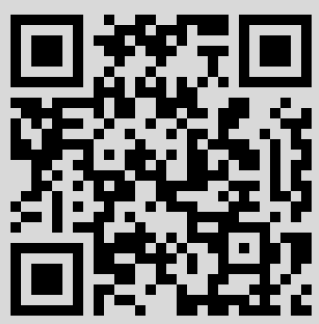




\section{НЕКОММУТАТИВНЫЕ ДЕФОРМАЦИИ КВАНТОВЫХ ТЕОРИЙ ПОЛЯ, ЛОКАЛЬНОСТЬ И ПРИЧИННОСТЬ}

Исследуются некоммутативные деформации квантовых теорий поля для различных звездочных произведений. Особое внимание уделяется локальным свойствам и регулярности деформированных полей. С использованием методов функционального анализа описаны основные структурные свойства вакуумных средних звездочно модифицированных произведений полей и полевых коммутаторов. В качестве замены условия микропричинности введено понятие $\theta$-локальности, где $\theta$ - параметр некоммутативности. Проанализированы условия сходимости и непрерывности звездочных произведений и определена алгебра функций, наиболее адекватная произведениям Мойала и Вика-Вороса. Эта алгебра соответствует концепции строгого деформационного квантования и является полезным средством при построении квантовой теории поля на некоммутативном пространстве-времени.

Ключевые слова: некоммутативная квантовая теория поля, звездочные произведения Мойала и Вика-Вороса, локальность, причинность, топологическая алгебра целых функций.

\section{1. ВВЕДЕНИЕ}

Интенсивное исследование моделей некоммутативной теории поля в последние годы мотивировано фундаментальными проблемами квантовой физики и теории гравитации [1]. Хотя идея некоммутативности пространства-времени достаточно стара и, как хорошо известно, восходит к Гейзенбергу и Снайдеру, развитие теории струн вызвало новую волну интереса к этой области исследований (см., например, обзор [2]). Почти все работы по этой тематике исходят из замены обычного поточечного произведения функций на пространстве-времени некоммутативным звездочным произведением. Как следствие, координатные функции удовлетворяют коммутационному соотношению

$$
\left[x^{\mu}, x^{\nu}\right]_{\star} \equiv x^{\mu} \star x^{\nu}-x^{\nu} \star x^{\mu}=i \theta^{\mu \nu},
$$

где $\theta^{\mu \nu}$ - вещественная антисимметричная матрица, играющая роль параметра некоммутативности и определяющая деформацию пространства Минковского. Одна-

*Физический институт им. П. Н. Лебедева РАН, Москва, Россия. E-mail: soloviev@lpi.ru 
ко эта деформация может быть объединена с основными принципами квантовой теории многими способами. Более того, имеется много звездочных произведений, совместных с коммутационным соотношением (1). Подавляющее большинство авторов использует звездочное произведение Мойала-Вейля-Грюневольда, но в некоторых недавних работах, например в статьях [3], [4], обсуждается теория поля с произведением Вика-Вороса (соответствующим нормальному упорядочению). Интересно, что при этом авторы приходят к противоположным выводам относительно эквивалентности теорий с различными звездочными произведениями.

$\mathrm{K}$ настоящему времени нет единой точки зрения на то, каков оптимальный путь построения самосогласованной некоммутативной теории поля. Много внимания уделяется изучению так называемой твистованной пуанкаре-ковариантности, нацеленной на восстановление пространственно-временных симметрий, нарушаемых некоммутативностью (см. статьи [5], [6] и ссылки в них). Вопрос о том, как именно следует реализовывать эту ковариантность, также является предметом споров, но во всех реализациях большую роль играет понятие твистованного тензорного произведения (которое мы предпочитаем называть скрученным), ассоциированного с заданным звездочным произведением. Ввиду отсутствия общепринятой формулировки некоммутативной квантовой теории поля вопрос о причинности наблюдаемых заслуживает дальнейшего исследования, поскольку он является решающим для физической интерпретации.

Следует отметить, что звездочные произведения и скрученные тензорные произведения можно рассматривать с двух различных точек зрения. Более распространено понимание их как формальных степенных рядов по параметру деформации $\theta$. Однако физически такой подход не вполне удовлетворителен. При анализе причинной структуры некоммутативных моделей следует учитывать, что этим произведениям внутренне присуща нелокальность, поскольку они определяются дифференциальными операторами бесконечного порядка. Для лучшего понимания их нелокальной структуры необходимо установить условия сходимости задающих формальных рядов и описать соответствующие топологические алгебры, в которых зависимость звездочных произведений от параметра некоммутативности является непрерывной. В дальнейшем мы придерживаемся второго подхода, который близок стратегии строгого деформационного квантования [7].

План изложения таков. Вначале мы напоминаем определения звездочных произведений Мойала и Вика-Вороса и ассоциированных с ними тензорных произведений. Затем показываем, что мойаловское скрученное произведение можно использовать для естественной деформации [8] квантовой теории поля, исходно заданной на коммутативном пространстве Минковского, и описываем основные свойства возникающей в результате некоммутативной теории поля. В частности, деформированные квантовые поля локализуемы в некоторых клиноподобных областях [9], но эта локализация существенно отличается от замены светового конуса световым клином, предлагавшейся ранее (см. статьи [10]-[12]) для теорий с пространственно-пространственной некоммутативностью, описывающих низкоэнергетический предел теории струн. Далее мы рассматриваем условия сходимости формальных степенных рядов, определяющих звездочные произведения, и формулируем основные свойства 
соответствующих функциональных алгебр [13]. Этот анализ и некоторые инструктивные примеры приводят к концепции $\theta$-локальности [14], которая, по нашему мнению, может использоваться для реализации причинности в некоммутативной теории поля.

\section{2. ЗВЕЗДОЧНЫЕ ПРОИЗВЕДЕНИЯ МОЙАЛА И ВИКА-ВОРОСА}

Формальные степенные ряды, задающие звездочные произведения Мойала и Вика-Вороса, записываются, соответственно, следующим образом:

$$
\begin{aligned}
\left(f \star_{\mathrm{M}} g\right)(x) & =f(x) \exp \left(\frac{i}{2} \overleftarrow{\partial_{\mu}} \theta^{\mu \nu} \overrightarrow{\partial_{\nu}}\right) g(x)= \\
& =f(x) g(x)+\sum_{n=1}^{\infty}\left(\frac{i}{2}\right)^{n} \frac{1}{n !} \theta^{\mu_{1} \nu_{1}} \ldots \theta^{\mu_{n} \nu_{n}} \partial_{\mu_{1}} \ldots \partial_{\mu_{n}} f(x) \partial_{\nu_{1}} \ldots \partial_{\nu_{n}} g(x)
\end{aligned}
$$

и

$$
\left(f \star_{\mathrm{V}} g\right)(x)=f(x) \exp \left(\frac{i}{2} \overleftarrow{\partial_{\mu}} \theta^{\mu \nu} \overrightarrow{\partial_{\nu}}+\frac{\vartheta}{2} \overleftarrow{\partial_{\mu}} \delta^{\mu \nu} \overrightarrow{\partial_{\nu}}\right) g(x)
$$

Во втором случае мы для простоты предполагаем, что матрица $\theta^{\mu \nu}$ имеет канонический блочно-диагональный вид с одним и тем же параметром $\vartheta>0$ в каждом $(2 \times 2)$-блоке $\left(\begin{array}{cc}0 & \vartheta \\ -\vartheta & 0\end{array}\right)$, хотя в приложениях различные блоки могут иметь различные $\vartheta$, включая и равные нулю. Эти два звездочных произведения играют центральную роль в подходе Вейля-Вигнера к квантовой механике, поскольку мойаловское произведение согласовано с симплектической структурой линейного фазового пространства и оба произведения согласованы с его комплексной структурой. Легко видеть, что эти произведения приводят к одному и тому же коммутационному соотношению (1) для координатных функций. Имеется простое соотношение между ними, а именно

$$
T\left(f \star_{\mathrm{M}} g\right)=T(f) \star_{\mathrm{V}} T(g), \quad \text { где } \quad T=e^{\vartheta \Delta / 4}, \quad \Delta=\sum_{\mu=1}^{d} \partial_{\mu}^{2} .
$$

Это соотношение соблюдается в смысле формальных степенных рядов и обсуждалось, в частности, в книге [15] в контексте квантовой механики. Однако с точки зрения строгого деформационного квантования произведения (2), (3) и оператор $T$ корректно определены только при довольно сильных ограничениях на функции. В формализме квантовой теории поля обычно используется пространство Шварца $S\left(\mathbb{R}^{d}\right)$, состоящее из гладких быстроубывающих функций. Принято считать, что оно является алгеброй относительно мойаловского звездочного произведения. Стоит отметить, однако, что ряд (2), вообще говоря, расходится для функций из $S\left(\mathbb{R}^{d}\right)$. Действительно, используя классическую теорему Бореля (см., например, монографию [16]), можно построить функции $f, g \in S\left(\mathbb{R}^{d}\right)$ такие, что последовательность $\left.f\left(\overleftarrow{\partial_{\mu}} \theta^{\mu \nu} \overrightarrow{\partial_{\nu}}\right)^{n} g\right|_{x=x_{0}}, n=0,1, \ldots$, совпадет с любой заданной числовой последовательностью. Более того, легко показать [13], что ряд, определяющий $f \star_{\mathrm{M}} f$, не сходится 
в топологии пространства $S\left(\mathbb{R}^{d}\right)$ даже для гауссовой функции $f(x)=e^{-\gamma|x|^{2}}$, если $\gamma>1 / \vartheta$. Однако $\star_{\mathrm{M}}$-умножение корректно определено на пространстве аналитических функций, которое будет охарактеризовано в разделе 6 . Это пространство плотно в $S\left(\mathbb{R}^{d}\right)$, и $\star_{\mathrm{M}}$-умножение допускает единственное непрерывное продолжение на пространство Шварца; это продолжение записывается в терминах преобразования Фурье следующим образом:

$$
\left(f \star_{\mathrm{M}} g\right)(x)=\frac{1}{(2 \pi)^{2 d}} \iint \hat{f}(k) \hat{g}\left(k^{\prime}\right) e^{i\left(k+k^{\prime}\right) \cdot x-i k \theta k^{\prime} / 2} d k d k^{\prime},
$$

где $k \theta k^{\prime} \stackrel{\text { def }}{=} k_{\mu} \theta^{\mu \nu} k_{\nu}^{\prime}$ - симплектическое скалярное произведение на $\mathbb{R}^{d}$. Легко видеть, что $S\left(\mathbb{R}^{d}\right)$ является ассоциативной топологической алгеброй относительно произведения, определяемого формулой (5). Оператор $T=e^{\vartheta \Delta / 4}$ также имеет естественное продолжение на $S\left(\mathbb{R}^{d}\right)$, совпадающее с операцией умножения фурье-образов на функцию $e^{-\vartheta|k|^{2} / 4}$. Его образ $\operatorname{im} T$ состоит из целых аналитических функций, удовлетворяющих неравенствам

$$
|f(x+i y)| \leqslant C_{N}(1+|x|)^{-N} e^{|y|^{2} / \vartheta}, \quad N=0,1, \ldots
$$

Такие функции образуют алгебру относительно произведения Вика-Вороса, а точнее, относительно умножения

$$
\left(f \star_{\mathrm{V}} g\right)(x)=\frac{1}{(2 \pi)^{2 d}} \iint \hat{f}(k) \hat{g}\left(k^{\prime}\right) e^{i\left(k+k^{\prime}\right) \cdot x-\left(i k \theta k^{\prime}+\vartheta k k^{\prime}\right) / 2} d k d k^{\prime},
$$

где $k k^{\prime}=\sum_{\mu} k_{\mu} k_{\mu}^{\prime}$ - евклидово скалярное произведение на $\mathbb{R}^{d}$. Однако все пространство $S\left(\mathbb{R}^{d}\right)$ не является $\star_{\mathrm{V}}$-алгеброй.

ПрЕДЛОЖЕНИЕ. Звездочное умножение Вика-Вороса не допускает непрерывного продолжения на пространство Шварца.

ДоказАтЕльство. Обозначим через $u$ линейный функционал $f \rightarrow \int f(x) d x=$ $\hat{f}(0)$. Ясно, что $u \in S^{\prime}$. Для любой функции $f \in \operatorname{im} T$ мы имеем

$$
u\left(f \star_{\mathrm{V}} f\right)=\frac{1}{(2 \pi)^{d}} \int \hat{f}(k) \hat{f}(-k) e^{\vartheta|k|^{2} / 2} d k .
$$

Положим $\hat{f}(k)=e^{-\vartheta|k|^{2} / 4}$ и рассмотрим последовательность $\hat{f}_{n}(k)=e^{-|k|^{4} / n} \hat{f}(k)$, которая очевидно принадлежит $\operatorname{im} T$ и сходится к $\hat{f}$ в фурье-симметричном пространстве $S\left(\mathbb{R}^{d}\right)$. Предположим, что существует непрерывное продолжение умножения (6) на это пространство. Тогда найдется функция $g \in S\left(\mathbb{R}^{d}\right)$ такая, что $g=f \star_{\mathrm{V}} f$ и $u\left(f_{n} \star_{\mathrm{V}} f_{n}\right) \rightarrow \hat{g}(0)$. Однако

$$
u\left(f_{n} \star_{\mathrm{V}} f_{n}\right)=\frac{1}{(2 \pi)^{d}} \int e^{-n|k|^{4} / 2} d k \rightarrow \infty
$$


и это противоречие завершает доказательство.

Аналогичные замечания могут быть сделаны и относительно скрученного тензорного произведения. В мойаловском случае оно определяется формулой

$$
\left(f_{1} \otimes_{\mathrm{M}} \cdots \otimes_{\mathrm{M}} f_{n}\right)\left(x_{1}, \ldots, x_{n}\right)=\prod_{1 \leqslant a<b \leqslant n} e^{i \partial_{x_{a}} \theta \partial_{x_{b}} / 2} f_{1}\left(x_{1}\right) \ldots f_{n}\left(x_{n}\right),
$$

где

$$
\partial_{x_{a}} \theta \partial_{x_{b}}=\frac{\partial}{\partial x_{a}^{\mu}} \theta^{\mu \nu} \frac{\partial}{\partial x_{b}^{\nu}}
$$

и часто называется "звездочным произведением в разных точках". Само звездочное произведение получается из скрученного тензорного отождествлением этих точек,

$$
\left(f_{1} \star_{\mathrm{M}} \cdots \star_{\mathrm{M}} f_{n}\right)(x)=\left.\left(f_{1} \otimes_{\mathrm{M}} \cdots \otimes_{\mathrm{M}} f_{n}\right)\right|_{x_{1}=\cdots=x_{n}=x}
$$

в полной аналогии с обычным поточечным произведением, которое получается тем же отождествлением из обычного тензорного произведения функций. В случае произведения Вика-Вороса мы имеем

$$
\left(f_{1} \otimes_{\mathrm{V}} \cdots \otimes_{\mathrm{V}} f_{n}\right)\left(x_{1}, \ldots, x_{n}\right)=\prod_{1 \leqslant a<b \leqslant n} e^{(i / 2) \partial_{x_{a}} \theta \partial_{x_{b}}+(\vartheta / 2) \partial_{a} \partial_{b}} f_{1}\left(x_{1}\right) \ldots f_{n}\left(x_{n}\right),
$$

где

$$
\partial_{a} \partial_{b}=\sum_{\mu} \frac{\partial}{\partial x_{a}^{\mu}} \frac{\partial}{\partial x_{b}^{\mu}}
$$

Скрученное тензорное произведение ассоциативно и может быть обобщено на функции нескольких переменных:

$$
\left(f \otimes_{\mathrm{M}} g\right)\left(x_{1}, \ldots, x_{m} ; x_{1}^{\prime}, \ldots, x_{n}^{\prime}\right)=\prod_{a=1}^{m} \prod_{b=1}^{n} e^{(i / 2) \partial_{x_{a}} \theta \partial_{x_{b}^{\prime}}} f\left(x_{1}, \ldots, x_{m}\right) g\left(x_{1}^{\prime}, \ldots, x_{n}^{\prime}\right)
$$

где правая часть определяется требованием согласованности с формулой (7) при $f=f_{1} \otimes_{\mathrm{M}} \cdots \otimes_{\mathrm{M}} f_{m}, g=g_{1} \otimes_{\mathrm{M}} \cdots \otimes_{\mathrm{M}} g_{n}$. Произведение $\otimes_{\mathrm{M}}$ корректно определено на достаточно гладких функциях и допускает непрерывное продолжение на пространства Шварца. В терминах преобразования Фурье соотношение между мойаловским и обычным тензорными произведениями принимает простой вид

$$
\left(\widehat{f \otimes_{\mathrm{M}}} g\right)\left(k, k^{\prime}\right)=e^{-i k \theta k^{\prime} / 2}(\hat{f} \otimes \hat{g})\left(k, k^{\prime}\right), \quad f, g \in S\left(\mathbb{R}^{d}\right)
$$

Участвующий здесь экспоненциальный множитель является мультипликатором для $S\left(\mathbb{R}^{2 d}\right)$ и определяет автоморфизм этого пространства. Аналогичное соотношение

3 Теоретическая и математическая физика, т. 163, № 3, 2010 г. 
соблюдается и для $f_{1} \otimes_{\mathrm{M}} \cdots \otimes_{\mathrm{M}} f_{n}$ с мультипликатором

$$
\mu_{n}(k)=\prod_{1 \leqslant a<b \leqslant n} e^{-i k_{a} \theta k_{b} / 2}
$$

Отсюда и из теоремы Шварца о ядре вытекает, что для каждого полилинейного непрерывного функционала $v: \underbrace{S\left(\mathbb{R}^{d}\right) \times \cdots \times S\left(\mathbb{R}^{d}\right)}_{n} \rightarrow \mathbb{C}$ найдется единственный линейный функционал $w \in S^{\prime}\left(\mathbb{R}^{n d}\right)$ такой, что диаграмма

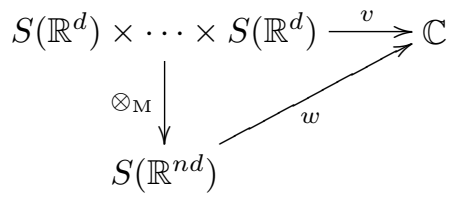

коммутативна.

\section{3. ТВИСТ-ДЕФОРМИРОВАННЫЕ КВАНТОВЫЕ ПОЛЯ}

Теперь мы перейдем к некоммутативной деформации [8], [9] квантовых теорий поля, которая может быть ассоциирована с мойаловским тензорным произведением. Эта конструкция не использует лагранжеву формулировку и является модельно-независимой, однако для простоты мы ограничимся здесь рассмотрением случая одного скалярного поля $\phi(x)$. Нашей исходной точкой является квантовая теория поля на коммутативном пространстве Минковского с обычными предположениями [17], [18] релятивистской ковариантности, локальности и физической стабильности, выраженной спектральным условием. Мы делаем также обычные предположения относительно области определения и непрерывности поля. Тогда каждое вакуумное среднее корректно определено как распределение умеренного роста,

$$
\left\langle\Psi_{0}, \phi\left(f_{1}\right) \ldots \phi\left(f_{n}\right) \Psi_{0}\right\rangle=\left(w^{(n)}, f_{1} \otimes \cdots \otimes f_{n}\right), \quad w^{(n)} \in S^{\prime}\left(\mathbb{R}^{4 n}\right)
$$

Идея состоит в том, чтобы деформировать эти распределения, заменив тензорные произведения их аргументов скрученными тензорными произведениями:

$$
\left(w_{\theta}^{(n)}, f_{1} \otimes \cdots \otimes f_{n}\right) \stackrel{\text { def }}{=}\left(w^{(n)}, f_{1} \otimes_{\mathrm{M}} \cdots \otimes_{\mathrm{M}} f_{n}\right)
$$

Из сказанного выше ясно, что существует единственный функционал $w_{\theta}^{(n)} \in S^{\prime}\left(\mathbb{R}^{4 n}\right)$, удовлетворяющий соотношению (13), и согласно теореме реконструкции Вайтмана последовательность деформированных распределений $w_{\theta}^{(n)}$ определяет некоторую теорию поля. Следует отметить, что на самом деле нет необходимости прибегать здесь к теореме реконструкции, поскольку нетрудно прямо определить квантовое 
поле $\phi_{\theta}$, вакуумные средние которого совпадают с деформированными вайтмановскими функциями $w_{\theta}^{(n)}$. А именно, теорема Шварца о ядре придает точный смысл векторам вида

$$
\Phi_{n}(f)=\int \phi\left(x_{1}\right) \ldots \phi\left(x_{n}\right) f\left(x_{1}, \ldots, x_{n}\right) \Psi_{0} d x_{1} \ldots d x_{n}
$$

где $\Psi_{0}$ - вакуумное состояние, а $f$ пробегает пространство $S\left(\mathbb{R}^{d n}\right)$. Следовательно, мы можем взять линейную оболочку $D$ всех таких векторов в качестве области определения исходного поля $\phi$. Для каждой функции $g \in S\left(\mathbb{R}^{4}\right)$ определим $\phi_{\theta}(g)$ формулами

$$
\phi_{\theta}(g) \Psi_{0}=\phi(g) \Psi_{0}, \quad \phi_{\theta}(g) \Phi_{n}(f)=\Phi_{n+1}\left(g \otimes_{M} f\right), \quad n \geqslant 1,
$$

с продолжением по линейности. Свойства полей $\phi_{\theta}$ можно суммировать [8] следующим образом.

Теорема 1. Пусть $\phi$ - эрмитово скалярное поле, удовлетворяющее всем ваймановским аксиомам, и пусть $w^{(n)}$ - его вайтмановские функиии. Тогда дебормированные поля $\phi_{\theta}$ корректно определены как операторнозначные распределения умеренного роста в гилъбертовом пространстве поля ф с той же областъю определения $и$

$$
\left\langle\Psi_{0}, \phi_{\theta}\left(g_{1}\right) \ldots \phi_{\theta}\left(g_{n}\right) \Psi_{0}\right\rangle=\left(w^{(n)}, g_{1} \otimes_{\mathrm{M}} \cdots \otimes_{\mathrm{M}} g_{n}\right), \quad g_{j} \in S\left(\mathbb{R}^{4}\right) .
$$

Вакуумное состояние $\Psi_{0}$ поля ф является ииклическим вектором для каждого поля $\phi_{\theta}$. Для этих полей соблюдается условие эрмитовости

$$
\phi_{\theta}(g)^{*} \supset \phi_{\theta}(\bar{g}), \quad g \in S\left(\mathbb{R}^{4}\right),
$$

и их вакуумные средние $w_{\theta}^{(n)}$ удовлетворяют спектральному условию и условию положительной определенности

$$
\sum_{m, n=0}^{N}\left(w_{\theta}^{(m+n)}, f_{m}^{*} \otimes f_{n}\right) \geqslant 0
$$

где $w_{\theta}^{(0)}=1, f_{0} \in \mathbb{C}, f^{*}\left(x_{1}, \ldots, x_{m}\right) \stackrel{\text { def }}{=} \overline{f\left(x_{m}, \ldots, x_{1}\right)} u\left\{f_{j}\right\}_{1 \leqslant j \leqslant N}-$ произвольный конечный набор пробных функций такой, что $f_{j} \in S\left(\mathbb{R}^{4 j}\right)$.

ДокАзАтЕльство. При любых $g \in S\left(\mathbb{R}^{4}\right), f \in S\left(\mathbb{R}^{4 n}\right)$ и $h \in S\left(\mathbb{R}^{4 m}\right)$

$$
\left\langle\Phi_{m}(h), \Phi_{n+1}\left(g \otimes_{\mathrm{M}} f\right)\right\rangle=\left\langle\Phi_{m+1}\left(\bar{g} \otimes_{\mathrm{M}} h\right), \Phi_{n}(f)\right\rangle
$$


или, эквивалентно,

$$
\left(w^{(m+n+1)}, h^{*} \otimes\left(g \otimes_{\mathrm{M}} f\right)\right)=\left(w^{(m+n+1)},\left(\bar{g} \otimes_{\mathrm{M}} h\right)^{*} \otimes f\right) .
$$

В самом деле, из определения (9) следует, что $\left(\bar{g} \otimes_{\mathrm{M}} h\right)^{*}=h^{*} \otimes_{\mathrm{M}} g$, поскольку матрица $\theta$ антисимметрична. Далее,

$$
\begin{aligned}
& \hat{h}^{*} \otimes\left(\widehat{g \otimes_{\mathrm{M}} f}\right)=\left(\hat{h}^{*} \otimes \hat{g} \otimes \hat{f}\right) e^{-(i / 2) k \theta\left(\sum_{a=1}^{n} p_{a}\right)}, \\
& \left(\widehat{h^{*} \otimes_{\mathrm{M}}} g\right) \otimes \hat{f}=\left(\hat{h}^{*} \otimes \hat{g} \otimes \hat{f}\right) e^{-(i / 2)\left(\sum_{b=1}^{m} q_{b}\right) \theta k},
\end{aligned}
$$

где $k$ - аргумент функции $\hat{g}, p_{a}$ - аргументы функции $\hat{f}$ и $q_{b}-$ аргументы функции $\hat{h}^{*}$. Снова используя антисимметричность $\theta$, мы приходим к соотношению (20), поскольку $\widehat{w}^{(m+n+1)}\left(q_{1}, \ldots, q_{m}, k, p_{1}, \ldots, p_{n}\right)$ содержит множитель $\delta\left(k+\sum_{b=1}^{m} q_{b}+\sum_{a=1}^{n} p_{a}\right)$, выражающий трансляционную инвариантность функционала $w^{(m+n+1)}$. Из (19) вытекает, что если линейная комбинация $\Phi$ векторов вида (14) равна нулю, то и $\phi_{\theta}(g) \Phi=0$, т. е. операторы $\phi_{\theta}(g)$ корректно определены. Очевидно также, что $\phi_{\theta}(g) \Phi \rightarrow 0$ при $g \rightarrow 0$ в $S\left(\mathbb{R}^{4}\right)$. Кроме того, из (19) следует $(17)$.

Тождество (16) вытекает непосредственно из определения (15). Если вектор $\Psi$ таков, что $\left\langle\Psi, \phi_{\theta}\left(g_{1}\right) \ldots \phi_{\theta}\left(g_{n}\right) \Psi_{0}\right\rangle=0$ при любых $g_{1}, \ldots, g_{n} \in S\left(\mathbb{R}^{4}\right)$ и всяком $n$, то $\left\langle\Psi, \Phi_{n}(f)\right\rangle=0$ для каждого вектора $\Phi_{n}(f)$ вида $(14)$, поскольку $\left\langle\Psi, \Phi_{n}(f)\right\rangle$ есть в точности тот элемент пространства $S^{\prime}\left(\mathbb{R}^{4 n}\right)$, который связан с полилинейной формой $\left(g_{1}, \ldots, g_{n}\right) \rightarrow\left\langle\Psi, \phi_{\theta}\left(g_{1}\right) \ldots \phi_{\theta}\left(g_{n}\right) \Psi_{0}\right\rangle$ диаграммой $(11)$. Следовательно, $\Psi=0$ и тем самым линейная оболочка векторов $\phi_{\theta}\left(g_{1}\right) \ldots \phi_{\theta}\left(g_{n}\right) \Psi_{0}$ плотна в гильбертовом пространстве. Ясно, что $\widehat{w}_{\theta}^{(n)}=\mu_{n} \cdot \widehat{w}^{(n)}$, где $\mu_{n}$ определяется формулой $(10)$, и поэтому деформированные вайтмановские функции имеют те же спектральные свойства, что и функции $w^{(n)}$. Для доказательства свойства (18) заметим, что $\left(w_{\theta}^{(m+n)}, f_{m}^{*} \otimes f_{n}\right)=\left(w^{(m+n)}, g_{m}^{*} \otimes_{\mathrm{M}} g_{n}\right)$, где $\hat{g}_{m}=\mu_{m} \cdot \hat{f}_{m}, \hat{g}_{n}=\mu_{n} \cdot \hat{f}_{n}$. Кроме того, в силу антисимметричности матрицы $\theta$ и трансляционной инвариантности $w^{(m+n)}$ мы имеем $\left(w^{(m+n)}, h \otimes_{\mathrm{M}} g\right)=\left(w^{(m+n)}, h \otimes g\right)$ при любых $h \in S\left(\mathbb{R}^{d m}\right)$ и $g \in S\left(\mathbb{R}^{d n}\right)$, ибо $\widehat{h \otimes_{\mathrm{M}} g}=(\hat{h} \otimes \hat{g}) e^{-(i / 2)\left(\sum_{b=1}^{m} q_{b}\right) \theta\left(\sum_{a=1}^{n} p_{a}\right)}$. Теорема доказана.

Согласно стандартной аргументации [17] любая другая реализация теории поля с вакуумными средними (16) и с циклическим трансляционно-инвариантным вакуумным состоянием унитарно эквивалентна построенной нами. Если исходное поле $\phi$ свободно, то его операторы рождения и уничтожения деформируются следующим образом:

$$
a_{\theta}(p)=e^{(i / 2) p \theta P} a(p), \quad a_{\theta}^{*}(p)=e^{-(i / 2) p \theta P} a^{*}(p),
$$

где $P$ - оператор энергии-импульса. Соответственно, они удовлетворяют деформированным каноническим коммутационным соотношениям (CCR)

$$
\begin{aligned}
& a_{\theta}(p) a_{\theta}\left(p^{\prime}\right)=e^{-i p \theta p^{\prime}} a_{\theta}\left(p^{\prime}\right) a_{\theta}(p), \quad a_{\theta}^{*}(p) a_{\theta}^{*}\left(p^{\prime}\right)=e^{-i p \theta p^{\prime}} a_{\theta}^{*}\left(p^{\prime}\right) a_{\theta}^{*}(p), \\
& a_{\theta}(p) a_{\theta}^{*}\left(p^{\prime}\right)=e^{i p \theta p^{\prime}} a_{\theta}^{*}\left(p^{\prime}\right) a_{\theta}(p)+2 \omega_{\mathbf{p}} \delta\left(\mathbf{p}-\mathbf{p}^{\prime}\right) .
\end{aligned}
$$


Твистованная CCR-алгебра (21) обсуждалась с разных точек зрения в работах [6], [19]-[22]. Как уже было сказано, эта деформация сохраняет трансляционную инвариантность, но она очевидным образом нарушает лоренц-инвариантность. Полный закон преобразования деформированных полей относительно собственной группы Пуанкаре выглядит следующим образом:

$$
U(a, \Lambda) \phi_{\theta}(f) U^{-1}(a, \Lambda)=\phi_{\Lambda \theta \Lambda^{\mathrm{T}}}\left(f_{a, \Lambda}\right),
$$

где $f_{(a, \Lambda)}(x)=f\left(\Lambda^{-1}(x-a)\right),(a, \Lambda) \in \mathcal{P}_{+}^{\uparrow}$. Следовательно, каждое поле $\phi_{\theta}$ преобразуется ковариантно лишь при тех лоренцевых преобразованиях, которые не меняют матрицу $\theta^{\mu \nu}$.

Рассматриваемая деформация приводит также к потере локальности, и поля $\phi_{\theta}$ не удовлетворяют стандартному условию микропричинности. Это легко увидеть, рассмотрев матричные элементы

$$
M_{\Phi}\left(x, x^{\prime}\right)=\left\langle\Psi_{0},\left[\phi_{\theta}(x), \phi_{\theta}\left(x^{\prime}\right)\right] \Phi\right\rangle
$$

в простейшем случае свободного поля. Если мы возьмем в качестве $\Phi$ нормированное двухчастичное состояние

$$
\Phi=\phi^{(-)}(h) \phi^{(-)}(h) \Psi_{0}, \quad \text { где } \quad h \in S\left(\mathbb{R}^{d}\right),
$$

то простое прямое вычисление показывает, что матричный элемент (23) не равен нулю для некоторых пространственноподобно разделенных точек. Более того, как показано в статье [8], это распределение не может обращаться в нуль ни в одной открытой области, т. е. $\operatorname{supp} M_{\Phi}=\mathbb{R}^{2 \cdot 4}$. Причина в том, что его преобразование Фурье имеет вид

$$
\widehat{M}_{\Phi}=-4 i \widehat{w}(k) \widehat{w}\left(k^{\prime}\right) \hat{h}(k) \hat{h}\left(k^{\prime}\right) \sin \frac{k \theta k^{\prime}}{2}
$$

где $\widehat{w}(k)$ - двухточечная функция поля $\phi$ в импульсном представлении. Следовательно, носитель распределения $\widehat{M}_{\Phi}$ содержится в произведении $\overline{\mathbb{V}}_{+} \times \overline{\mathbb{V}}_{+}$двух замкнутых верхних конусов. Множитель $(\hat{h} \otimes \hat{h}) \sin \left(k \theta k^{\prime} / 2\right)$ не исчезает на множестве $\operatorname{supp}(\widehat{w} \otimes \widehat{w})$, если $\Phi \neq 0$. Поэтому $M_{\Phi}$ является граничным значением нетривиальной аналитической функции. Такое распределение не может обращаться в нуль на непустом открытом множестве по обобщенной теореме единственности для аналитических функций (см. книгу [18], теорема В.7). Таким образом, мы можем заключить, что некоммутативная деформация путем перехода в вакуумных средних к скрученным тензорным произведениям приводит к конфликту между спектральным условием и принципом причинности.

\section{4. ЛОКАЛИЗАЦИЯ В КЛИНОПОДОБНЫХ ОБЛАСТЯХ}

Деформированные поля $\phi_{\theta}$ с различными $\theta$ сохраняют некоторые свойства относительной локализации, установленные в работах [9], [21]. В этих работах отмечено, что каждой антисимметричной матрице $\theta$ можно естественным образом сопоставить 
клиноподобную область $W_{\theta}$ в пространстве-времени. Если матрица имеет стандартный вид

$$
\theta_{1}=\left(\begin{array}{cccc}
0 & \vartheta_{e} & 0 & 0 \\
-\vartheta_{e} & 0 & 0 & 0 \\
0 & 0 & 0 & \vartheta_{m} \\
0 & 0 & -\vartheta_{m} & 0
\end{array}\right), \quad \vartheta_{e} \geqslant 0
$$

то ассоциированный с ней клин $W_{1}$ определяется неравенством $x^{1}>\left|x^{0}\right|$. Стационарная подгруппа матрицы $\theta_{1}$ относительно действия $\theta \rightarrow \Lambda \theta \Lambda^{\mathrm{T}}$ собственной группы Лоренца $\mathcal{L}_{+}$совпадает со стационарной подгруппой клина $W_{1}$ относительно действия $W \rightarrow \Lambda W$, поэтому имеется взаимно однозначное соответствие между орбитами $\theta_{1}$ и $W_{1}$. В работах [9], [21] показано, что поля $\phi_{\theta}$ удовлетворяют следующему соотношению клинолокальной коммутативности: если множества $\operatorname{supp} f+W_{\theta}$ и $\operatorname{supp} g+W_{\theta^{\prime}}$ пространственноподобно разделены, то $\left[\phi_{\theta}(f), \phi_{\theta^{\prime}}(g)\right] \Psi=0$ для всех $\Psi \in D$. Поэтому деформированные поля следует представлять себе как объекты, локализуемые в клиноподобных областях, а не в точках пространства-времени.

Такая локализация похожа на изучавшуюся в работе [23] в рамках алгебраической квантовой теории поля. Как отмечается в статьях [9], [21], даже эта слабая форма локальной коммутативности позволяет построить некоторую теорию рассеяния. Однако этот тип локализации радикально отличается от замены светового конуса световым клином, которая предлагалась ранее в качестве возможной модификации условия микропричинности в теории поля с пространственно-пространственной некоммутативностью, соответствующей низкоэнергетическому пределу теории струн. В следующем разделе обсуждается инструктивный пример, дающий некоторое представление о такой модификации.

\section{5. ДЕФОРМИРОВАННЫЙ ВИКОВ КВАДРАТ СВОБОДНОГО ПОЛЯ}

Рассмотрим нормально упорядоченный квадрат свободного скалярного поля в четырехмерном пространстве-времени, заменив обычное произведение в его определении мойаловским звездочным произведением,

$$
\begin{aligned}
\mathcal{O}(x) & \stackrel{\text { def }}{=}: \phi \star_{\mathrm{M}} \phi:(x)=\lim _{x_{1}, x_{2} \rightarrow x}: \phi\left(x_{1}\right) \phi\left(x_{2}\right):+ \\
& +\sum_{n=1}^{\infty}\left(\frac{i}{2}\right)^{n} \frac{1}{n !} \theta^{\mu_{1} \nu_{1}} \ldots \theta^{\mu_{n} \nu_{n}} \lim _{x_{1}, x_{2} \rightarrow x}: \partial_{\mu_{1}} \ldots \partial_{\mu_{n}} \phi\left(x_{1}\right), \partial_{\nu_{1}} \ldots \partial_{\nu_{n}} \phi\left(x_{2}\right): .
\end{aligned}
$$

Легко видеть, что в случае пространственно-пространственной некоммутативности, когда $\theta^{23}=-\theta^{32}=\vartheta \neq 0$, а остальные элементы матрицы $\theta$ нулевые, коммутатор $[\mathcal{O}(x), \mathcal{O}(y)]$ исчезает в клине, определяемом неравенством $\left|x^{0}-y^{0}\right|<\left|x^{1}-y^{1}\right|$. В работе [24] показано, что аналогичный коммутатор с производной по времени 
отличен от нуля в некоторых точках вне этого клина, а именно

$$
\left.\left\langle 0\left|\left[\mathcal{O}(x), \partial_{0} \mathcal{O}(y)\right]_{-}\right| p_{1}, p_{2}\right\rangle\right|_{x^{0}=y^{0}} \neq 0
$$

В указанной работе выдвинута гипотеза, что это верно и в общем случае при наличии в наблюдаемых производных по времени. Более тщательное рассмотрение [8] показывает, что пространственно-пространственная некоммутативность нарушает условие микропричинности даже для наблюдаемых без производных по времени. Более того, коммутатор $[\mathcal{O}(x), \mathcal{O}(y)]$ отличен от нуля для любых точек $\bar{x}$ и $\bar{y}$, лежащих вне клина $\left|x^{0}-y^{0}\right|<\left|x^{1}-y^{1}\right|$. Говоря совсем точно, имеется состояние $\Phi$ такое, что пара $(\bar{x}, \bar{y})$ принадлежит носителю распределения $\left\langle\Psi_{0},[\mathcal{O}(x), \mathcal{O}(y)] \Phi\right\rangle$. Звездочный коммутатор

$$
[\mathcal{O}(x), \mathcal{O}(y)]_{\star}=\mathcal{O}(x) \star_{\mathrm{M}} \mathcal{O}(y)-\mathcal{O}(y) \star_{\mathrm{M}} \mathcal{O}(x)
$$

также обсуждался в литературе с противоречащими друг другу заключениями. Анализ его матричных элементов, выполненный в той же работе [8], показывает, что он отличен от нуля всюду. Эти результаты показывают, в частности, что разложения матричных элементов по степеням параметра некоммутативности не сходятся в топологии пространства распределений умеренного роста, поскольку частичные суммы этих разложений очевидно удовлетворяют условию микропричинности.

Этот простой пример демонстрирует также, что некоммутативная теория поля, использующая звездочное произведение Вика-Вороса, более нелокальна, чем теория с мойаловским произведением. Вычисление, аналогичное выполненному в статье [8], показывает, что в случае произведения Вика-Вороса матричный элемент соответствующего коммутатора между вакуумом и двухчастичным состоянием (24) имеет вид

$$
\begin{aligned}
\left\langle\Psi_{0},\left[\mathcal{O}_{\mathrm{V}}(x), \mathcal{O}_{\mathrm{V}}(y)\right] \Phi\right\rangle= & 8 \iiint \epsilon\left(k^{0}\right) \delta\left(k^{2}-m^{2}\right) e^{\vartheta \mathbf{k}\left(\mathbf{p}_{2}-\mathbf{p}_{1}\right) / 2} e^{-i k \cdot(x-y)-i p_{1} \cdot x-i p_{2} \cdot y} \times \\
& \times \prod_{a=1}^{2} \theta\left(p_{a}^{0}\right) \delta\left(p_{a}^{2}-m^{2}\right) \cos \left(\frac{1}{2} k \theta p_{a}\right) \hat{h}\left(p_{a}\right) \frac{d k d p_{1} d p_{2}}{(2 \pi)^{12}}
\end{aligned}
$$

где $\mathbf{k}=\left(k^{2}, k^{3}\right)$. Это выражение содержит дополнительный экспоненциальный множитель $e^{\vartheta \mathbf{k}\left(\mathbf{p}_{2}-\mathbf{p}_{1}\right) / 2}$ по сравнению с мойаловским случаем. По этой причине оно не является распределением умеренного роста и корректно определено в координатном представлении лишь на аналитических пробных функциях.

\section{6. СХОДИМОСТЬ ЗВЕЗДОЧНЫХ ПРОИЗВЕДЕНИЙ И АДЕКВАТНЫЕ ФУНКЦИОНАЛЬНЫЕ ПРОСТРАНСТВА}

Перейдем теперь к условиям сходимости звездочных произведений и описанию пространств пробных функций [13], [14], наиболее адекватных некоммутативной теории поля. Как сказано выше, представляющие произведения Мойала и Вика-Вороса 
формальные степенные ряды расходятся, вообще говоря, для функций из пространства Шварца. Однако они сходятся при дополнительном условии вида

$$
(1+|x|)^{N}\left|\partial^{\kappa} f(x)\right|<C_{N} B^{|\kappa|} \sqrt{\kappa !},
$$

где $C_{N}$ и $B$ - константы, зависящие от $f$, и

$$
B<\frac{1}{\sqrt{|\theta|}}, \quad|\theta|=\max \left|\theta^{\mu \nu}\right| .
$$

В формуле (26) используются следующие мультииндексные обозначения: $\kappa=$ $\left(\kappa_{1}, \ldots, \kappa_{d}\right) \in \mathbb{Z}_{+}^{d}, \partial^{\kappa}=\partial_{x_{1}}^{\kappa_{1}} \ldots \partial_{x_{d}}^{\kappa_{d}},|\kappa|=\kappa_{1}+\cdots+\kappa_{d}, \kappa !=\kappa_{1} ! \ldots \kappa_{d} !$. Сходимость произведения Мойала при указанном условии доказана в работах [13], [14] двумя различными способами. Однако функциональное пространство, определяемое неравенствами (26), не является алгеброй даже относительно обычного поточечного произведения. Чтобы получить алгебру, мы берем функции, удовлетворяющие аналогичным неравенствам со сколь угодно малым $B$. Пространство таких функций обозначаем через $\mathscr{S}^{1 / 2}$. Его естественная топология задается системой норм

$$
\|f\|_{B, N}=\sup _{x, \kappa}(1+|x|)^{N} \frac{\left|\partial^{\kappa} f(x)\right|}{B^{|\kappa|} \sqrt{\kappa !}} .
$$

Легко видеть, что $\mathscr{S}^{1 / 2}$ является пространством Фреше, т. е. оно метризуемо и полно. Более того, оно ядерно, что следует из результата статьи [25], в которой устанавлена ядерность пространств Гельфанда-Шилова $S^{\beta}$. Однако следует подчеркнуть, что $\mathscr{S}^{1 / 2} \neq S^{1 / 2}$. Пространство $S^{1 / 2}$ представляет собой индуктивный предел семейства пространств $S^{1 / 2, B}$ при $B \rightarrow \infty$ (и, в частности, оно неметризуемо), тогда как $\mathscr{S}^{1 / 2}$ является проективным пределом того же семейства при $B \rightarrow 0$. Пространства $S^{\beta}$ с $\beta<1 / 2$ предлагались для использования в некоммутативной квантовой теории поля в статье [26]. Свойство ядерности является решающим при выводе теоремы Шварца о ядре, которая играет важную роль в аксиоматической формулировке квантовой теории поля. Простое доказательство аналогичной теоремы для широкого класса пространств, включающего $\mathscr{S}^{1 / 2}$ и $S^{\beta}$, дано в работах [27], [28]. Следующий результат развивает теорему 6 работы [14].

ТЕорема 2. Пространство $\mathscr{S}^{1 / 2}\left(\mathbb{R}^{d}\right)$ является топологической алгеброй как относительно звездочного произведения Мойала, так и относительно произведения Вика-Вороса. Если $f, g \in \mathscr{S}^{1 / 2}\left(\mathbb{R}^{d}\right)$, то ряды, представляющие эти произведения, абсолютно сходятся в этом пространстве. Более того, эти произведения непрерьвны в нем по параметру некоммутативности $\theta$, и оператор $T=e^{\vartheta \Delta / 4}$ является автоморбизмом данного пространства.

ДоказАтельство. Сначала мы охарактеризуем те дифференциальные операторы

$$
\sum_{\lambda \in \mathbb{Z}_{+}^{d}} c_{\lambda} \partial^{\lambda}
$$


бесконечного порядка, которые действуют непрерывно на $\mathscr{S}^{1 / 2}$. Предположим, что $\sum_{\lambda} c_{\lambda} z^{\lambda}$ имеет рост не выше экспоненциального с порядком 2 и типом $b$, что эквивалентно ограничению

$$
\left|c_{\lambda}\right| \leqslant C(2 b)^{|\lambda| / 2} \frac{1}{\sqrt{\lambda !}} .
$$

Применяя оператор $(28)$ к функции $f \in \mathscr{S}^{1 / 2}$, мы получаем

$$
\begin{aligned}
(1+|x|)^{N}\left|\partial^{\kappa} \sum_{\lambda} c_{\lambda} \partial^{\lambda} f(x)\right| & \leqslant\|f\|_{B, N} \sum_{\lambda} c_{\lambda} B^{|\kappa+\lambda|} \sqrt{(\kappa+\lambda) !} \leqslant \\
& \leqslant\|f\|_{B, N} 2^{|\kappa| / 2} B^{|\kappa|} \sqrt{\kappa !} \sum_{\lambda} c_{\lambda} 2^{|\lambda| / 2} B^{|\lambda|} \sqrt{\lambda !} .
\end{aligned}
$$

Предположим также, что

$$
b<\frac{1}{4 B^{2}}
$$

Тогда ряд в правой части (30) сходится и, взяв $B^{\prime} \geqslant B \sqrt{2}$, мы получаем

$$
\left\|\sum_{\lambda} c_{\lambda} \partial^{\lambda} f\right\|_{B^{\prime}, N} \leqslant C^{\prime}\|f\|_{B, N} .
$$

Следовательно, оператор (28) при любом $b<\infty$ отображает пространство $\mathscr{S}^{1 / 2}$ в себя непрерывным образом. Заметим, что тип роста оператора в определении (2)

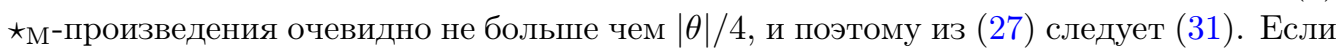
матрица $\theta$ имеет стандартный вид, указанный в разделе 2 , то $b$ есть просто $\vartheta$. Мы заключаем, что $T=e^{\vartheta \Delta / 4}$ является автоморфизмом пространства $\mathscr{S}^{1 / 2}\left(\mathbb{R}^{d}\right)$.

Далее, операторы, входящие в определения (7) и (8), являются автоморфизмами пространства $\mathscr{S}^{1 / 2}\left(\mathbb{R}^{n d}\right)$, и ряды, представляющие скрученные тензорные произведения, абсолютно сходятся в $\mathscr{S}^{1 / 2}\left(\mathbb{R}^{n d}\right)$. Используя формулу Лейбница, легко проверить, что $\mathscr{S}^{1 / 2}$ является топологической алгеброй относительно поточечного произведения. Следовательно, отображение $\mathscr{S}^{1 / 2}\left(\mathbb{R}^{2 d}\right) \rightarrow \mathscr{S}^{1 / 2}\left(\mathbb{R}^{d}\right): f(x, y) \rightarrow f(x, x)$ непрерывно, поскольку это именно то линейное отображение, которое ассоциировано с поточечным умножением, и поскольку $\mathscr{S}^{1 / 2}\left(\mathbb{R}^{2 d}\right)=\mathscr{S}^{1 / 2}\left(\mathbb{R}^{d}\right) \widehat{\otimes} \mathscr{S}^{1 / 2}\left(\mathbb{R}^{d}\right)$ по теореме о ядре. Звездочные произведения (2), (3) Мойала и Вика-Вороса получаются из $f \otimes_{\mathrm{M}} g$ и $f \otimes_{\mathrm{V}} g$ посредством этого отображения и поэтому также непрерывны. Их непрерывность по $\theta$ может быть установлена тем же способом, что использовался при доказательстве теоремы 5 работы [13]. Теорема доказана.

Существенно, что $\mathscr{S}^{1 / 2}$ есть наибольшее из подпространств пространства Шварца $S$, обладающих свойствами, установленными теоремой 2. Однако, чтобы точно описать нелокальность, обусловленную некоммутативностью, недостаточно использовать лишь пространство $\mathscr{S}^{1 / 2}$. Для лучшего понимания причинной структуры некоммутативных моделей полезно глубже исследовать свойства убывания полевых 
коммутаторов. Простой и хорошо известный способ описания свойств убывания распределений состоит в рассмотрении их свертки с достаточно быстро убывающими пробными функциями. Однако функции, удовлетворяющие установленному выше условию сходимости, не могут убывать слишком быстро. Наиболее подходящее пространство состоит из функций, убывающих по гауссову закону. Точнее говоря, разумно использовать двухпараметрическое семейство пространств $S_{1 / 2, A}^{1 / 2, B}$, каждое из которых состоит из функций с конечной нормой

$$
\|f\|_{A, B}=\sup _{\kappa, x} e^{|x / A|^{2}} \frac{\left|\partial^{\kappa} f(x)\right|}{B^{|\kappa|} \sqrt{\kappa !}} .
$$

В приложении к работе [8] показано, что это пространство нетривиально при $A B>2$ и тривиально при $A B<\sqrt{2}$.

\section{7. $\theta$-ЛОКАЛЬНОСТЬ}

Используя быстроубывающие пробные функции, можно показать, что некоторые свойства причинности соблюдаются независимо от типа некоммутативности. С этой целью опять рассмотрим деформированный нормально упорядоченный квадрат $\mathcal{O}(x)=: \phi \star \phi:(x)$ свободного поля, на сей раз усредняя его с функциями данного типа:

$$
\mathcal{O}\left(f_{a}\right)=\int \mathcal{O}(x) f(x-a) d x, \quad f \in S_{1 / 2, A}^{1 / 2, B} .
$$

Требуется оценить асимптотическое поведение коммутатора $\left[\mathcal{O}\left(f_{a}\right), \mathcal{O}\left(g_{-a}\right)\right]$ двух усредненных наблюдаемых при большом пространственноподобном разделении. Зафиксируем пространственноподобный вектор $a$ и обозначим через $\gamma$ его угловое расстояние до светового конуса,

$$
\gamma=\inf _{\xi^{2} \geqslant 0}\left|\xi-\frac{a}{|a|}\right|
$$

Вычисляя матричный элемент

$$
\left\langle\Psi_{0},\left[\mathcal{O}\left(f_{a}\right), \mathcal{O}\left(g_{-a}\right)\right] \Phi\right\rangle
$$

между вакуумом и двухчастичным состоянием $\Phi=\phi^{(-)}\left(h_{1}\right) \phi^{(-)}\left(h_{2}\right) \Psi_{0}$, мы находим [8], что с увеличением $|a|$ он убывает гауссовым образом, а именно

$$
\left|\left\langle\Psi_{0},\left[\mathcal{O}\left(f_{a}\right), \mathcal{O}\left(g_{-a}\right)\right] \Phi\right\rangle\right| \leqslant C_{\Phi, A^{\prime}}\|f\|_{A, B}\|g\|_{A, B} e^{-2\left|\gamma a / A^{\prime}\right|^{2}}
$$

при любом $A^{\prime}>A$. Из условия сходимости $B<1 / \sqrt{|\theta|}$ и условия нетривиальности $A B>2$ следует, что наилучший результат получается при $A \sim \sqrt{|\theta|} . \quad$ В итоге мы 
заключаем, что при пространственноподобном разделении, стремящемся к бесконечности, матричный элемент (32) убывает как $e^{-|\gamma a|^{2} /|\theta|}$. Этот результат справедлив как в случае произведения Мойала, так и в случае произведения Вика-Вороса. Более того, можно показать, что любой матричный элемент коммутатора имеет аналогичное поведение.

Оценка (33) вполне информативна, но она может быть выражена в более абстрактных терминах, даже более удобных для приложений. Это можно сделать, используя еще один класс функциональных пространств, которые могут быть сопоставлены конусоподобным областям и определяются следующим образом. Пусть $U-$ конус в $\mathbb{R}^{d}$. Гладкая функция $f$ на $\mathbb{R}^{d}$ принадлежит пространству $S^{1 / 2, B}(U)$, если она удовлетворяет условию

$$
\sup _{x \in U}(1+|x|)^{N}\left|\partial^{\kappa} f(x)\right|<C_{N} B^{|\kappa|} \sqrt{\kappa !} .
$$

Оказывается, результат (33) эквивалентен тому, что при любом $B<1 / \sqrt{|\theta|}$ матричный элемент, рассматриваемый как обобщенная функция, имеет непрерывное продолжение на пространство $S^{1 / 2, B}(\mathbb{V})$, ассоциированное с относительным световым конусом $\mathbb{V}=\left\{(x, y) \in \mathbb{R}^{4} \times \mathbb{R}^{4}:(x-y)^{2} \geqslant 0\right\}$.

Это подсказывает, как условие микропричинности может быть обобщено на квантовые поля на некоммутативном пространстве-времени. Нелокальные эффекты в некоммутативной теории поля определяются структурой звездочного произведения, и можно ожидать, что в этой теории все матричные элементы $\left\langle\Phi,\left[\phi(x), \psi\left(x^{\prime}\right)\right]_{-} \Psi\right\rangle$ полевых коммутаторов (или антикоммутаторов для ненаблюдаемых полей) допускают непрерывное продолжение на пространство $S^{1 / 2, B}(\mathbb{V})$, индекс $B$ которого по порядку величины равен $1 / \sqrt{|\theta|}$, но может зависеть, вообще говоря, от полей $\phi, \psi$ и от состояний $\Phi, \Psi$. Это условие было введено в работе [14] и для краткости названо $\theta$-локальностью. Следует подчеркнуть, что оно согласуется с пуанкаре-ковариантностью. Предположительно $\theta$-локальность выражает отсутствие нарушений причинности на масштабах, которые много больше фундаментальной длины $\sqrt{|\theta|}$. Если это действительно так, то сформулированное условие могло бы быть названо макропричинностью. Вполне возможно, что в физически реалистичных некоммутативных моделях матричные элементы полей являются распределениями умеренного роста. Однако и в этом случае пространство $S^{1 / 2, B}(\mathbb{V})$ может служить инструментом для формулировки причинности, и из сказанного выше ясно, что его роль может оказаться более существенной в случае звездочного произведения Вика-Вороса.

\section{8. ЗАКЛЮЧЕНИЕ}

Физические следствия некоммутативной квантовой теории поля, получаемой путем перехода к скрученным тензорным произведениям, нуждаются в дальнейшем исследовании. Мы не затронули здесь твистованную пуанкаре-ковариантность, но следует отметить, что наивное сочетание рассмотренной выше деформации с такой 
ковариантностью может привести к теории, которая физически эквивалентна недеформированной, аналогично теории, обсуждавшейся в работе [20]. Вопрос о том, как наилучшим образом объединить идею пространственно-временной некоммутативности с основными принципами квантовой физики, пока остается открытым. Условие $\theta$-локальности на интуитивном уровне означает, что коммутаторы наблюдаемых при большом пространственноподобном разделении ведут себя как $e^{-|x-y|^{2} / \theta}$, и это похоже на условие асимптотической коммутативности, использовавшееся ранее в нелокальной квантовой теории поля на коммутативном пространстве Минковского. Как показано в работе [29], асимптотическая коммутативность в сочетании с релятивистской ковариантностью и спектральным условием обеспечивает существование СРТ-симметрии и нормальную связь спина со статистикой для нелокальных полей. Это служит дополнительным аргументом использования $\theta$-локальности для формулировки причинности в некоммутативной квантовой теории поля. Пространство $\mathscr{S}^{1 / 2}$, являясь максимальной топологической алгеброй с абсолютной сходимостью относительно звездочного произведения, полностью адекватно концепции строгого деформационного квантования и может использоваться для формулировки и изучения некоммутативных моделей квантовой теории поля вне рамок теории возмущений.

Благодарности. Автор выражает свою благодарность Организационному комитету и проф. И. Я. Арефьевой за приглашение сделать доклад на Второй международной конференции "Струнная теория поля и смежные вопросы" (Москва, 2009 г.). Работа поддержана РФФИ (грант № 09-01-00835) и Программой поддержки ведущих научных школ (грант НШ-1615.2008).

\section{Список литературы}

[1] S. Doplicher, K. Fredenhagen, J. E. Roberts, Comm. Math. Phys., 172:1 (1995), 187-220.

[2] R. J. Szabo, Phys. Rep., 378 (2003), 207-299.

[3] S. Galluccio, F. Lizzi, P. Vitale, Phys. Rev. D, 78:8 (2008), 085007.

[4] A. P. Balachandran, M. Martone, Modern Phys. Lett. A, 24:22 (2009), 1721-1730.

[5] M. Chaichian, P. P. Kulish, K. Nshijima, A. Tureanu, Phys. Lett. B, 604:1-2 (2004), 98-102.

[6] M. Riccardi, R. J. Szabo, JHEP, 01 (2008), 016.

[7] M. A. Rieffel, Comm. Math. Phys., 122:4 (1989), 531-562.

[8] M. A. Soloviev, Phys. Rev. D, 77:12 (2008), 125013.

[9] H. Grosse, G. Lechner, JHEP, 09 (2008), 131.

[10] Yi Liao, K. Sibold, Phys. Lett. B, 549:3-4 (2002), 352-361.

[11] L. Álvarez-Gaumé, M. A. Vázquez-Mozo, Nucl. Phys. B, 668:1-2 (2003), 293-321.

[12] C.-S. Chu, K. Furuta, T. Inami, Internat. J. Modern Phys. A, 21:1 (2006), 67-82.

[13] М. А. Соловьев, ТМФ, 153:1 (2007), 3-17.

[14] M. A. Soloviev, J. Phys A, 40:48 (2007), 14593-14604.

[15] Ф. А. Березин, М. А. Шубин, Уравнение Шредингера, Изд-во МГУ, М., 1983.

[16] Л. Хермандер, Анализ линейных дифберенциальных операторов с частными производными. Т. 1. Теория распределений и анализ Фуръе, Мир, М., 1986.

[17] Р. Стритер, А. С. Вайтман, РCT, спин и статистика и все такое, Наука, М., 1966.

[18] Н. Н. Боголюбов, А.А. Логунов, А. И. Оксак, И. Т. Тодоров, Общие принципы квантовой теории поля, Наука, М., 1987. 
[19] A. P. Balachandran, T. R. Govindarajan, G. Mangano, A. Pinzul, B. A. Qureshi, S. Vaidya, Phys. Rev. D, 75:4 (2007), 045009.

[20] G. Fiore, J. Wess, Phys. Rev. D, 75:10 (2007), 105022.

[21] H. Grosse, G. Lechner, JHEP, 11 (2007), 012.

[22] P. Aschieri, F. Lizzi, P. Vitale, Phys. Rev. D, 77:2 (2008), 025037.

[23] J. Mund, B. Schroer, J. Yngvason, Comm. Math. Phys., 268:3 (2006), 621-672.

[24] O. W. Greenberg, Phys. Rev. D, 73:4 (2006), 045014.

[25] Б. С. Митягин, Труды ММО, 9 (1960), 317-328.

[26] M. Chaichian, M. N. Mnatsakanova, A. Tureanu, Yu. A. Vernov, JHEP, 09 (2008), 125.

[27] A. G. Smirnov, M. A. Soloviev, On kernel theorems for Fréchet and DF spaces, arXiv: math.FA/0501187.

[28] A. G. Smirnov, Integral Transforms Spec. Funct., 20:3-4 (2009), 309-318.

[29] М. А. Соловьев, ТМФ, 121:1 (1999), 139-164. 